\title{
Gastrin stimulates pancreatic cancer cell directional migration by activating the Ga12/13-RhoA-ROCK signaling pathway
}

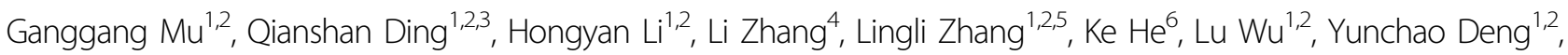
Dongmei Yang ${ }^{1,2}$, Lianlian $\mathrm{Wu}^{1,2}$, Ming $\mathrm{Xu}^{1,2}$, Jie Zhou ${ }^{1,2}$ and Honggang $\mathrm{Yu}^{1,2}$

\begin{abstract}
The mechanism by which gastrin promotes pancreatic cancer cell metastasis is unclear. The process of directing polarized cancer cells toward the extracellular matrix is principally required for invasion and distant metastasis; however, whether gastrin can induce this process and its underlying mechanism remain to be elucidated. In this study, we found that gastrin-induced phosphorylation of paxillin at tyrosine 31/118 and RhoA activation as well as promoted the metastasis of PANC-1 cancer cells. Depletion of Ga12 and Ga13 inhibited the phosphorylation of paxillin and downstream activation of GTP-RhoA, blocked the formation and aggregation of focal adhesions and facilitated polarization of actin filaments induced by gastrin. Suppression of RhoA and ROCK also exhibited identical results. Selective inhibition of the CCKBR-Ga12/13-RhoA-ROCK signaling pathway blocked the reoriented localization of the Golgi apparatus at the leading edge of migrated cancer cells. YM022 and Y-27632 significantly suppressed hepatic metastasis of orthotic pancreatic tumors induced by gastrin in vivo. Collectively, we demonstrate that gastrin promotes Golgi reorientation and directional polarization of pancreatic cancer cells by activation of paxillin via the CCKBR-Ga12/13-RhoA-ROCK signal pathway.
\end{abstract}

\section{Introduction}

Pancreatic cancer is one of the most common malignancies and is a leading cause of cancer-related death worldwide $^{1}$. The incidence rate of pancreatic cancer continues to approximate the death rate, implying that most patients with pancreatic cancer die as a result of this cancer largely because it is highly aggressive and likely to metastasize $^{2}$. A better understanding of the mechanisms underlying pancreatic cancer metastasis is essential for exploring novel strategies to enhance the current treatment efficacy and improve the prognosis of patients.

Correspondence: Honggang Yu (yuhonggang1968@hotmail.com)

'Department of Gastroenterology, Renmin Hospital of Wuhan University, Wuhan 430060, China

${ }^{2}$ Key Laboratory of Hubei Province for Digestive System Disease, Renmin Hospital of Wuhan University, Wuhan, China

Full list of author information is available at the end of the article

These authors contribute equally: Ganggang Mu, Qianshan Ding, Hongyan Li.
Directional cell migration is required for many important physiological processes, such as embryonic development, immune surveillance, and wound healing ${ }^{3,4}$. Additionally, directional cell migration plays a key role in pivotal steps that promote tumor metastasis, such as cellular migration and invasion into the surrounding stroma $^{5,6}$. During directional cell migration, cancer cells acquire a highly polarized phenotype (with membrane protrusion and a retracting tail), form focal adhesions and reorient the Golgi apparatus to move proteins to specific intracellular locations ${ }^{7,8}$.

Cholecystokinin B receptor (CCKBR), a member of the family of G protein-coupled receptors (GPCR), couples with gastrin and cholecystokinin, which are principally expressed in the gastrointestinal tract ${ }^{9}$. CCKBR was first regarded as a regulator of gastric acid secretion and the calcium signaling pathway, and now CCKBR has been 
identified and characterized as a stimulator in multiple malignancies, including pancreatic cancer ${ }^{9-11}$. Compared with normal tissues, the expression level of CCKBR is significantly increased in pancreatic cancerous tissues ${ }^{12}$. The human pancreas produces gastrin during fetal development, and no gastrin is expressed in the healthy adult pancreas; however, gastrin is reexpressed in pancreatic cancerous tissues, where it enhances proliferation and migration through an autocrine mechanism ${ }^{11,13}$. However, the role of CCKBR in pancreatic cancer metastasis still remains to be clarified.

The Rho family of small GTPases, including RhoA, Rac1, Cdc42, and Rab43, exerts important functions in cancer progression by affecting multiple aspects, such as promoting cytoskeletal reorganization, intracellular trafficking, and Golgi orientation ${ }^{14,15}$. It has been reported that following gastrin binding, activated CCKBR undergoes a conformational change that exchanges GDP for

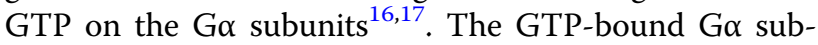
unit then interacts with downstream signaling effectors, resulting in the activation of various second messenger molecules that are responsible for eliciting cellular responses ${ }^{16,17}$. On the other hand, paxillin is one of the most important proteins in focal adhesion formation and is essential for cellular adhesion, motility, and invasion ${ }^{18}$. In highly aggressive tumors, high levels of phosphorylated paxillin indicate a stronger ability to migrate and metastasize $^{18}$. Previous work by others and ourselves showed that gastrin can induce rapid phosphorylation of paxillin ${ }^{19,20}$. Inspired by these findings, we hypothesize that gastrin/CCKBR may trigger the activation of RhoA and paxillin, induce directional cell migration, and in turn, promote metastasis of pancreatic cancer cells.

In this study, we showed that by co-ordinating paxillin activation and Golgi apparatus reorientation, gastrin plays a crucial role in the acquisition of a polarized phenotype and, accordingly, in directional cell migration of PANC-1 cells. Furthermore, during these events, activation of Go12/13-RhoA-ROCK signaling is a pivotal mechanism. Thus, our findings elucidate a potential explanation for the tumor microenvironment in modulating the directional migration of pancreatic cancer cells at the molecular level.

\section{Materials and methods}

\section{Antibodies and reagents}

Antibodies were obtained from the following commercial sources: anti-paxillin monoclonal, anti-paxillin $\mathrm{p}$ Tyr31 polyclonal, and anti-paxillin p-Tyr118 polyclonal (Invitrogen, CA, USA); anti-RhoA monoclonal, anti-FAK polyclonal, anti- $\beta$-actin polyclonal, and goat anti-mouse IgG, F(ab')2-TRITC (Santa Cruz, CA, USA); goat antirabbit IgG $(\mathrm{H}+\mathrm{L}), \mathrm{F}\left(\mathrm{ab}^{\prime}\right) 2$ Fragment (Alexa Fluor ${ }^{\circledR} 555$ Conjugate) antibody (Cell Signaling Technology, USA).
Horseradish peroxidase-labeled antibodies were purchased from Thermo Pierce (Rockford, USA). Gastrin and Y-27632 were obtained from Sigma-Aldrich (St. Louis, USA). Rhosin was obtained from Calbiochem ${ }^{\circledR}$ (La Jolla, CA, Germany). Acti-stain ${ }^{\mathrm{Tx}} 488$ Fluorescent Phalloidin and Rho Activation Assay Biochem Kit were obtained from Cytoskeleton, Inc. (Japan). 4',6-Diamidine-2'-phenylindole dihydrochloride (DAPI) was obtained from Roche Diagnostics (Japan).

\section{Cell culture}

The human pancreatic PANC-1 cancer cells, obtained from the Cell Bank of the Chinese Academy of Sciences (Shanghai, China), were cultivated in Dulbecco's modified Eagle's medium (DMEM; Gibco, NY, USA) in a humidified incubator containing $5 \% \mathrm{CO}_{2}$ at $37^{\circ} \mathrm{C}$.

\section{Transfection experiments}

The synthetic small interfering MISSION esiRNA tar-

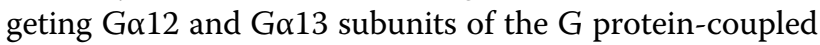
receptor family was obtained from Sigma-Aldrich Biotechnology. In brief, PANC-1 cells were treated with 200 $\mathrm{nM}$ gastrin for $1 \mathrm{~h}$ after transfection with $50 \mathrm{ng} / \mu \mathrm{l}$ of targeting siRNA for $48 \mathrm{~h}$. The depletion of specific proteins was analyzed by western blotting.

\section{Protein extraction and western blotting}

At the designated time points, cell protein extraction and protein concentration quantification were performed following the manufacturer's instructions. In brief, equal amounts of protein were separated on polyacrylamide gels and transferred to nitrocellulose membranes. The blots were incubated with $1 \mu \mathrm{g}$ of primary antibodies at $4{ }^{\circ} \mathrm{C}$ overnight. After incubation with horseradish peroxidaselinked secondary antibodies, the signals of target proteins were visualized by an ECL system (Millipore, CA).

\section{Transwell migration and invasion assays}

Cell migration and invasion were determined by Transwell ${ }^{\circledR}$ Inserts pre-coated with Matrigel (Corning, NY, USA). The tumor cells were incubated for $24 \mathrm{~h}$ and then the medium and cells remaining in the top chambers were removed. After fixing with methanol and staining with Hoechst 33342, the cells that penetrated the lowermembrane surface were counted by an inverted microscope. To assess invasion ability, filters were pre-coated with diluted Matrigel while the filters in the migration experiments were not pre-coated with Matrigel.

\section{Endogenous RhoA activity assay}

Active RhoA (GTP-bound) was captured using the Rho Activation Assay Biochem Kit (Cytoskeleton, Inc., Japan). PANC-1 cells that were grown in logarithmic phase in a $10-\mathrm{cm}$ plate were starved in serum-free medium 
containing Y-27632/Rhosin for $24 \mathrm{~h}$ and were subsequently stimulated with gastrin. Cells were lysed in a buffer containing $100 \mathrm{mM} \mathrm{NaCl}, 20 \mathrm{mM}$ Tris-HCL, $1 \%$
Triton X-100, $2 \mathrm{mM} \mathrm{NaF}$, and protease inhibitors (Roche Diagnostics). Cell extracts were homogenized and incubated overnight with GST-rhotekin-RBD fusion protein
$\mathbf{A}$

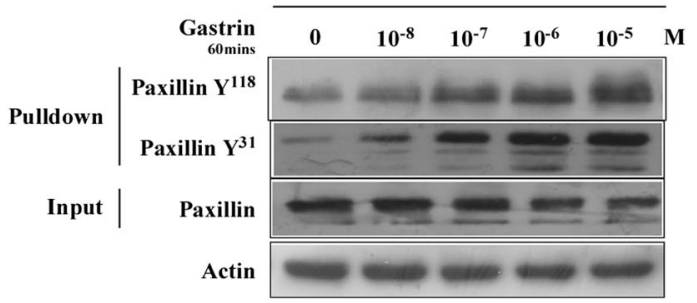

B

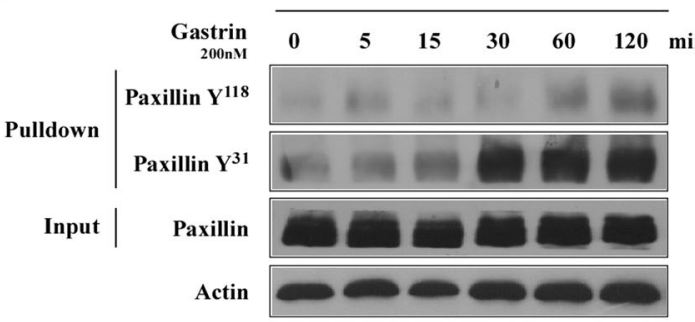

C

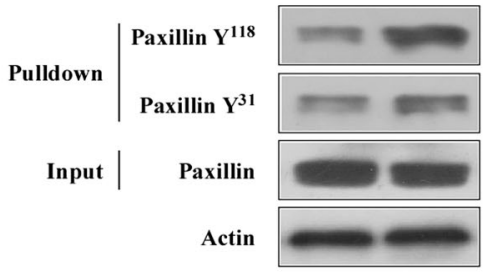

D

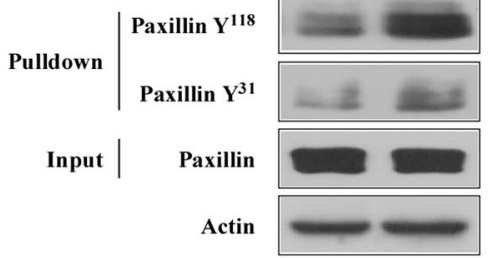

$\mathbf{E}$
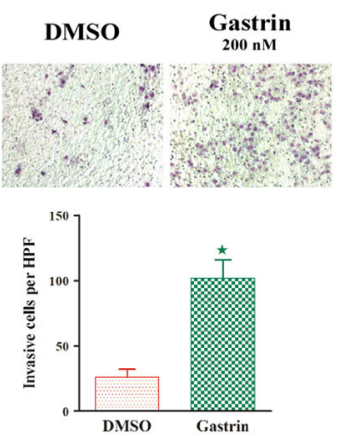

F

Migration
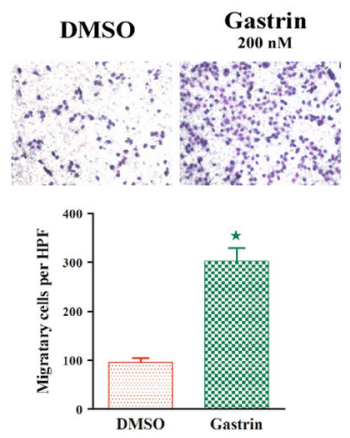

G

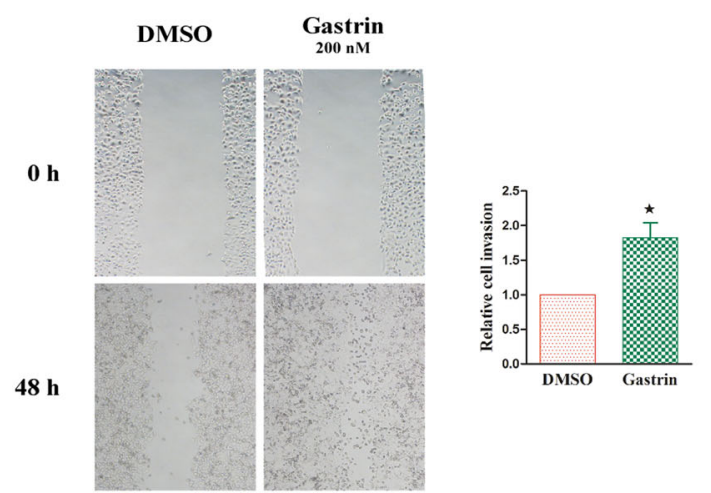

Fig. 1 Gastrin regulates paxillin activation and migration of pancreatic cancer cells. a Gastrin dose dependently induces elevated tyrosine phosphorylation of paxillin in PANC-1 cells. b Gastrin time dependently induces elevated tyrosine phosphorylation of paxillin in PANC-1 cells. c 200 nM gastrin treatment for 60 min can activate paxillin in HGC cells. $\mathbf{d} 200 \mathrm{nM}$ gastrin treatment for 60 min can activate paxillin in HT-29 cells. e, $\mathbf{f}$ Gastrin (200 nM) promotes the invasion (e) and migration (f) of PANC-1 cells, as indicated by Boyden chamber assays. $\mathbf{g}$ The wound healing assay indicates the elevated migration of PANC-1 cells following $48 \mathrm{~h}$ of gastrin stimulation. $\beta$-actin was used as a loading control. The values indicate the means \pm SD from three independent experiments 
(Cytoskeleton, Japan). Active GTP-RhoA in the lysates was evaluated by immunoblotting with a monoclonal RhoA antibody.

\section{Immunofluorescence assay}

For immunofluorescent staining, PANC-1 cells were seeded onto coverslips at $1 \times 10^{5}$ cells $/ \mathrm{ml}$ and were maintained to adhere to the slides. Cells were pre-treated with gastrin or DMSO for the indicated time, were fixed in preheated $3.7 \%$ paraformaldehyde (PFA) and permeabilized with $0.4 \%$ Triton X-100 for 5 min before blocking with goat serum in PBS. Afterward, cells were incubated with primary antibodies at $4{ }^{\circ} \mathrm{C}$ overnight and were visualized by fluorescent-labeled secondary antibodies as indicated. Finally, cells were embedded with $1 \mu \mathrm{g} / \mathrm{ml}$ of DAPI, and fluorescent images were acquired using an upright Olympus fluorescence microscope.

\section{Assay for Golgi orientation}

PANC-1 cells were treated with siRNA before seeding on sterile coverslips and were left to grow to confluence before wounding. Monolayers were wounded by scratching with 20-200 $\mu \mathrm{l}$ pipette tips and were washed three times with PBS. The wounded monolayer was treated with gastrin for the indicated time. For Golgi apparatus reorientation assays, the first row of cells facing wounding was determined. The Golgi apparatus, nucleus, and Factin were labeled by $\beta$-cop (red), DAPI (blue), and Acti$\operatorname{stain}^{\text {Tw }} 488$ Phalloidin reagent (green), respectively. Golgi orientation was evaluated for the first row of cells, and analysis was performed using Cell Scan software. As indicated in the schematic plot (Supplementary Figure 1), the dotted arrow denotes the vertical direction toward the scratched front, and the solid black arrow indicates the migrating direction of the cells. The vast majority of the Golgi apparatus $(>70 \%)$ within a $60^{\circ}$ angle facing the scratched line was considered as the positive orientation, while less than $70 \%$ Golgi apparatus in a $60^{\circ}$ angle or dispersed in cytoplasm was considered as the negative orientation. To calculate angles between the Golgi migrated direction ( $>70 \%$ Golgi apparatus migrating direction) and the $0^{\circ}$ vertical scratched line clockwise, $5^{\circ}$ is classified as one unit. For quantitative analysis, at least 100 cells and Golgi stacks were assessed per experiment, and data analysis was performed using Origin 9.1 software.

\section{Animal experiments and orthotopic implantation of pancreatic cancer cells}

All in vivo studies were approved by the Committee on the Use of Live Animals in Teaching and Research at Wuhan University. PANC-1 cells were harvested and resuspended into a single-cell suspension with PBS containing 0.1\% Matrigel (BD Biosciences). Then, suspensions containing $1 \times 10^{6}$ cells $/ 50 \mu \mathrm{l}$ were injected into the head or neck region of the pancreatic parenchyma of $\mathrm{BALB} / \mathrm{c}$ nude mice (6 weeks, female). All mice were killed on day 42, and the pancreatic tumors in situ were dissected and weighed. Isolated tumors were cut in half and one part was lysed for western blotting while the other part was formalin-fixed for immunohistochemical assay. Livers of the mice were also prepared for $\mathrm{H} \& \mathrm{E}$ staining to determine the level of metastasis.

\section{Statistical analysis}

For all cell experiments, the mean values \pm SD of at least triplicate experiments were calculated. Statistical significance between values of different experimental conditions was analyzed by Student's $t$ test. The value of $P<$ 0.05 was considered to indicate statistical significance.

\section{Results}

Gastrin phosphorylates and activates paxillin in a concentration- and time-dependent manner in pancreatic cancer cells

The phosphorylation of Tyr31 and Tyr118 sites is crucial for the activation of paxillin ${ }^{18}$. In this study, we observed that gastrin can phosphorylate Tyr31 and Tyr118 of paxillin in the PANC-1 pancreatic cancer cell line in a concentration-dependent manner. As shown in Fig. 1a, at a low concentration $\left(10^{-8} \mathrm{M}\right)$, gastrin can significantly increase the phosphorylation of both Tyr31 and Tyr118. As the concentration of gastrin increased, the level of phosphorylation was increased accordingly and reached a peak level at $10^{-7} \mathrm{M}$ and $10^{-6} \mathrm{M}$ for Tyr31 and Tyr118, respectively. No significant change in the total level of paxillin was observed.

The function of gastrin in paxillin phosphorylation is also time dependent. As shown in Fig. 1b, Tyr118 and Tyr31 began to show obvious phosphorylation after culturing with $200 \mathrm{nM}$ gastrin for $60 \mathrm{~min}$ and $15 \mathrm{~min}$, respectively, and the phosphorylation had positive relevance with the inducing time. Notably, the total expression level of paxillin was stable during these assays (Fig. 1b). Hence in the subsequent assays, we cultured the cells for $60 \mathrm{~min}$ in $200 \mathrm{nM}$ gastrin to activate paxillin. These experimental conditions were also verified in the gastric cancer cells HGC and the colon cancer cells HT29 (Fig. 1c, d).

\section{Gastrin promotes the vertical migration, vertical invasion, and horizontal movements of PANC-1 cells}

Previous studies have demonstrated that gastrin can promote the migration of many types of cancer cells ${ }^{10,11}$. Herein, we explored whether gastrin exerted similar effects on PANC-1 cells. We utilized Transwell chambers without or with the addition of Matrigel to evaluate vertical migration and invasion ability, respectively, and utilized the scratched wound healing assay in a culture dish 


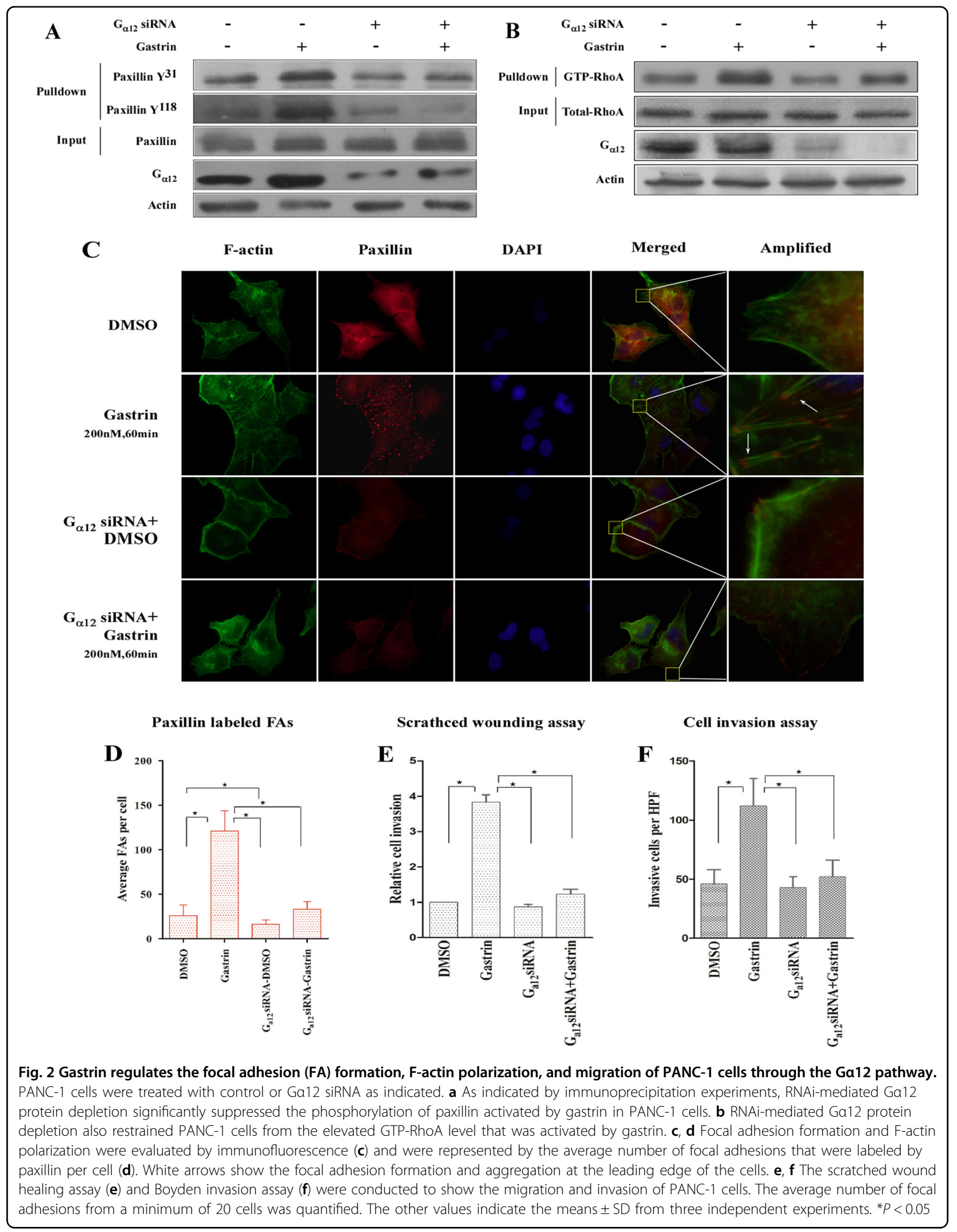




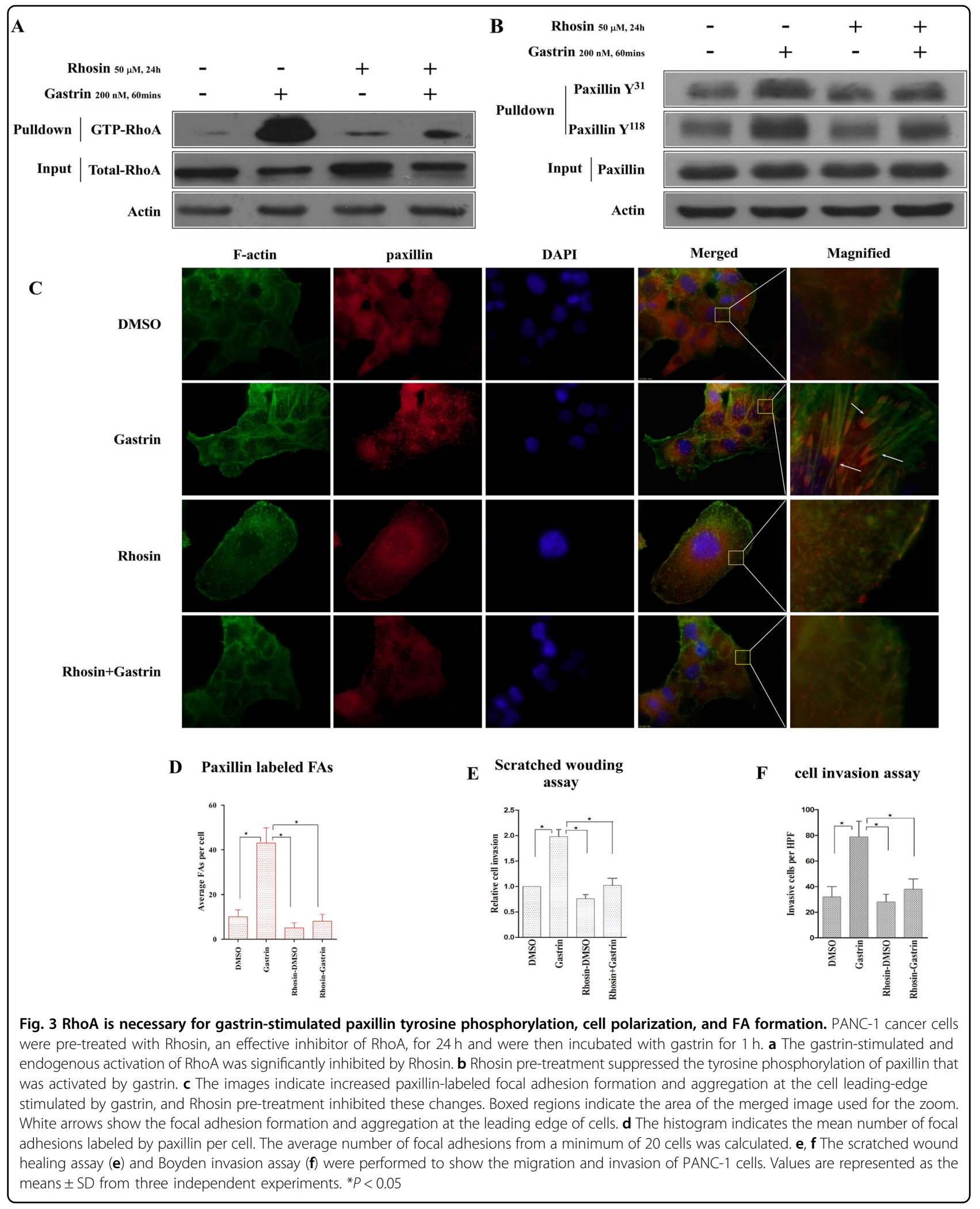




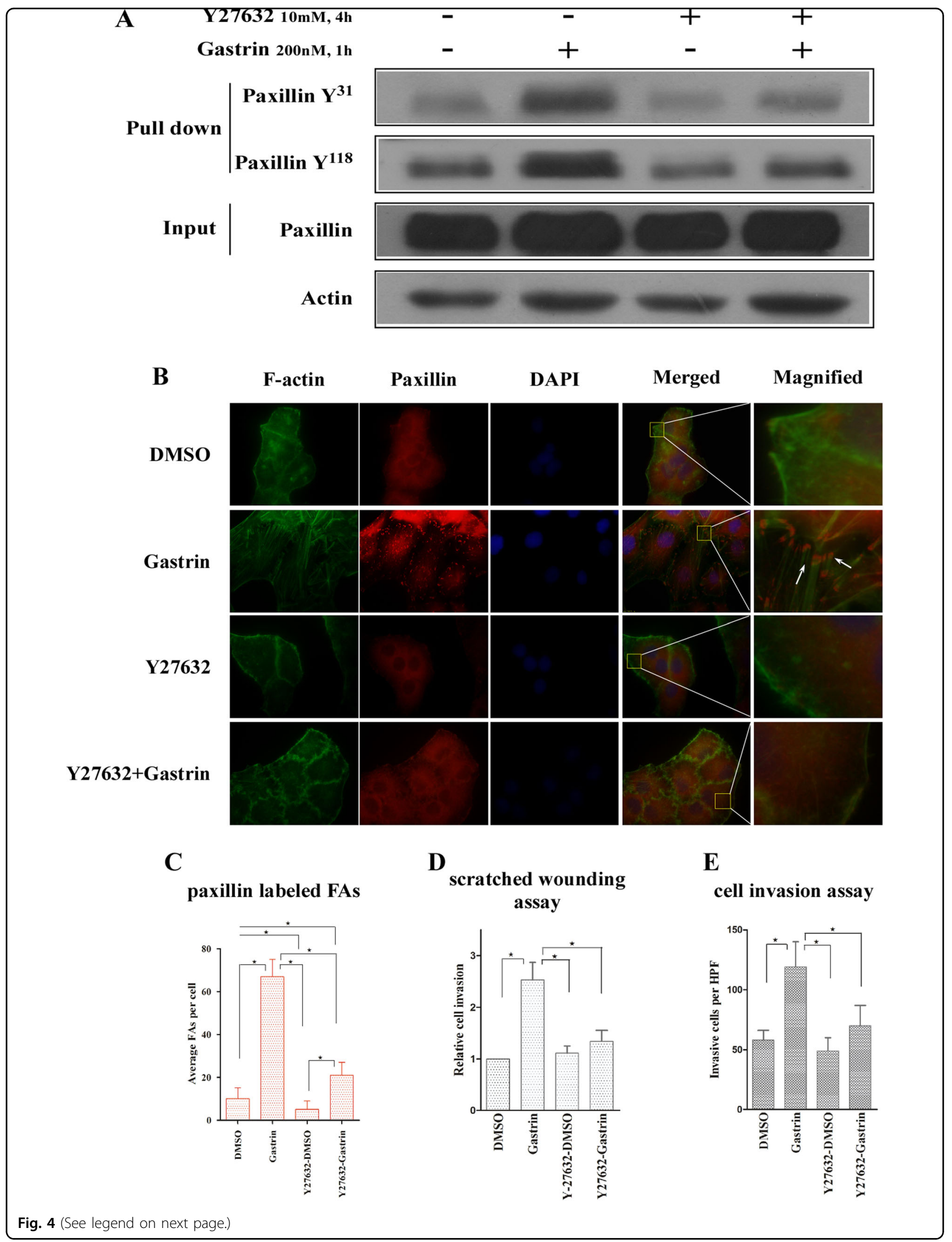


(see figure on previous page)

Fig. 4 Gastrin-regulated PANC-1 cell polarization, migration, and invasion through activation of ROCK. PANC-1 cancer cells were preincubated with Y27632 reagent, an effective inhibitor of ROCK, for $4 \mathrm{~h}$ and were then stimulated with gastrin for $1 \mathrm{~h}$. a Activated paxillin with high levels of phosphorylation at Tyr118 and Tyr31 that were induced by gastrin were significantly inhibited by Y27632 pre-treatment, while total paxillin was unchanged. $\mathbf{b}$ Immunofluorescence images indicate an increased polarization of F-actin and aggregation of focal adhesions at the leading edge in gastrin-treated PANC-1 cells. Y27632 pre-treatment obviously blocked the focal adhesion enrichment and cell polarization. Boxed regions represent the area of the merged image used for the zoom; c The mean number of large-sized focal adhesions labeled by paxillin per cell are indicated, and the average number of focal adhesions from a minimum of 20 cells was calculated. $\mathbf{d}$, e The migration and invasion of PANC-1 cells pre-treated with Y27632 were investigated by scratched wound healing (d) and the Boyden invasion assay (e). Values indicate the means \pm SD from three independent experiments. ${ }^{*} P<0.05$

covered with fibronectin to analyze horizontal movement. As shown in Fig. 1e-g, $200 \mathrm{nM}$ gastrin significantly enhanced the motility of PANC-1 cells.

\section{Gastrin activates GTP-RhoA in a time-dependent manner}

The Rho GTPase family is associated with actin cytoskeleton rearrangement, cell polarity, and cellular directional movement, and RhoA is one of the most crucial members of this family ${ }^{14}$. It was reported that gastrin can induce the activation of RhoA in colonic cancer ${ }^{11}$; however, it is unclear whether RhoA has a similar function in pancreatic cancer. Herein, we showed that with $200 \mathrm{nM}$ gastrin, the level of GTP-RhoA increased continuously with the culture time (Supplementary Figure 2). Our results indicate that gastrin can also promote the activation of RhoA in pancreatic cancer cells.

Gastrin phosphorylates paxillin, activates RhoA, modulates the formation of focal adhesions and cell polarization via its $\mathrm{G}$ protein-coupled receptor Ga12/13

To determine the underling mechanism by which gastrin activates paxillin and RhoA in pancreatic cancer, we began by investigating the gastrin receptor, CCKBR. The heterotrimeric $\mathrm{G}$ proteins $\mathrm{G \alpha} 12$ and $\mathrm{G \alpha} 13$ are widely expressed in human tissues ${ }^{17,21-23}$. We hypothesize that Go12/13 may be the crucial mediator in the function of gastrin in pancreatic cancer. After validating the knockdown effects of Ga12 siRNA, we used it to inhibit the expression of Go12 in PANC-1 cells. As shown in Fig. 2a, b, compared with the control group, G $\alpha 12$ inhibition significantly reduced the activation of paxillin and RhoA, and the effects of gastrin on stimulating paxillin and RhoA were partially or totally rescued when Go12 siRNA was transected. Immunofluorescence analysis further validated our western blot results. As shown in Fig. 2c, d, in the control (DMSO) group, paxillin, which is the marker protein of focal adhesions, was mainly located in the cytoplasm without clustering, and F-actin was also scattered and not bundling or polarized. After stimulation with gastrin, the numbers of focal adhesions were obviously increased with a larger area and more brightness, especially in the protruding lamellipodia, and F-actin was bundle-like in the same direction. After Go12 siRNA was added, phenomena such as focal adhesions, F-actin assembly, and cellular protrusion were all significantly reduced. The scratch wound healing assay and cell invasion assay were also conducted to validate that $G \alpha 12$ is crucial for gastrin's function: after Ga12 inhibition, the effects of gastrin were remarkably alleviated (Fig. 2e, f). In addition, we observed similar results using G $\alpha 13$ siRNA to treat the cell (Supplementary Figure 3A-F), and collectively, we demonstrated that CCKBR-Go12/13 were pivotal to transmit the signal from gastrin in pancreatic cancer.

\section{Gastrin promotes directional pancreatic cancer cell migration via RhoA/ROCK activation}

Others have established that RhoA and its downstream effector, ROCK, modulate cell polarization and cellular directional movement ${ }^{24-26}$. However, in pancreatic cancer, the roles of RhoA and ROCK in cell directional migration are not clear. After using the RhoA inhibitor Rhosin to block the RhoA-ROCK pathway (Fig. 3a), we noted that phosphorylation on Tyr31 and Tyr118 of paxillin was significantly reduced, although with gastrin induction (Fig. 3b). In immunofluorescence assays, we also observed that Rhosin significantly reduced the cellular polarization, focal adhesion formation, and F-actin assembly induced by gastrin (Fig. 3c, d) and promoted decreased wound healing and invasion ability of the cells (Fig. 3e, f). We also used an inhibitor of ROCK, Y27632, to treat the PANC-1 cells and obtain similar results (Fig. 4a-e). We also utilized another focal adhesion protein marker, FAK, and as expected, the results were in agreement (Supplementary Figures 4 and 5). Our results confirm that RhoA-ROCK is the main manipulator in activating paxillin and FAK and is crucial for cell directional migration induced by gastrin in pancreatic cancer.

\section{Gastrin induced Golgi apparatus reorientation via the CCKBR-Ga12/13-RhoA-ROCK pathway}

Reorientation of the secretory machinery is involved in directional cell migration ${ }^{8}$. This process is in coordination with rearrangement of the cytoskeleton and focal adhesion formation ${ }^{8}$. However, the molecular mechanisms that dominate this reorientation are not well 


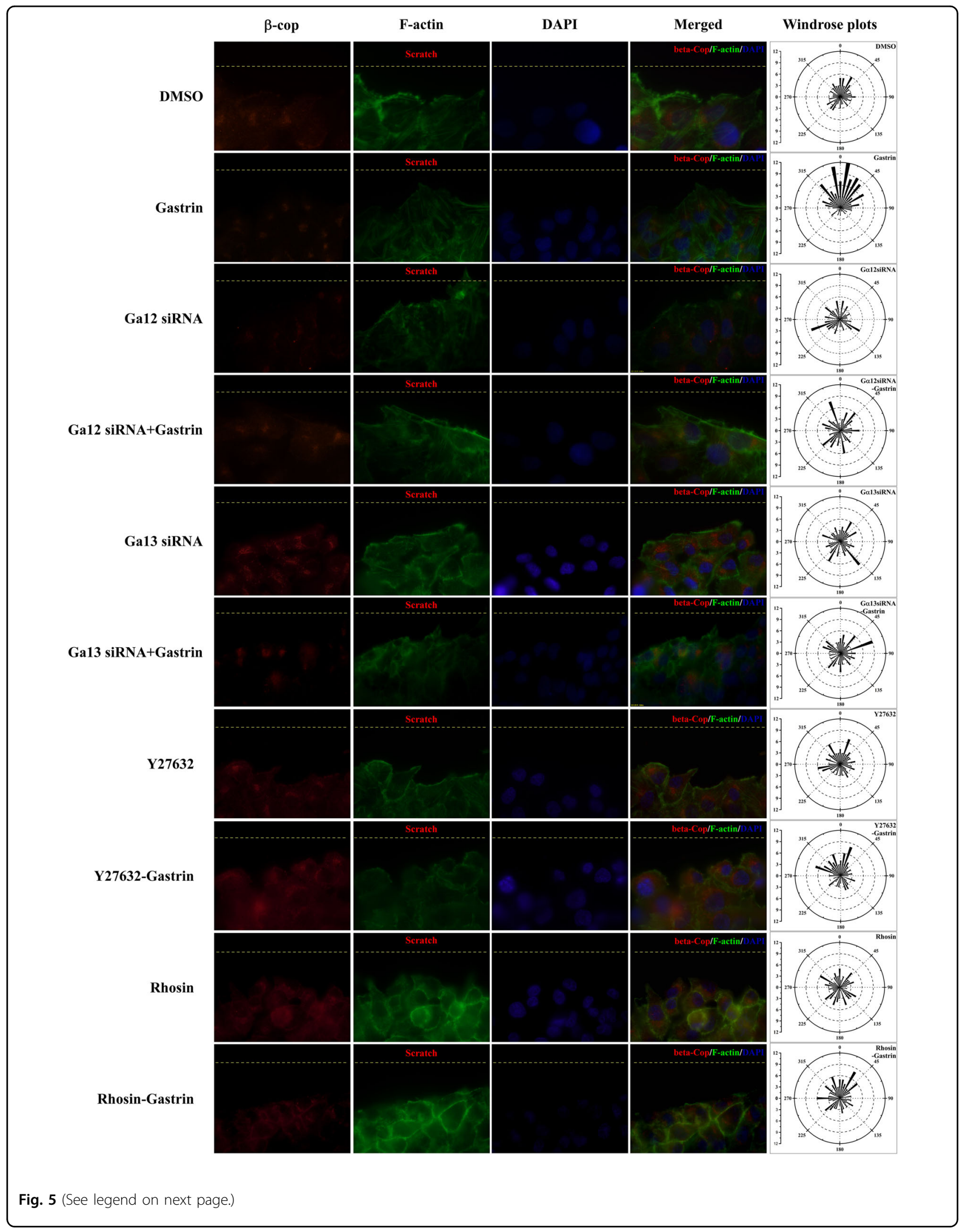


(see figure on previous page)

Fig. 5 Gastrin promotes Golgi aggregation and cell polarization at the leading edge in PANC-1 cells through activation of the Ga12/ 13-RhoA-ROCK signaling pathway. PANC-1 cells were allowed to grow to confluence for $24 \mathrm{~h}$ and were then pre-treated with Ga12 siRNA, Ga13 siRNA, Rosin, and Y27632 to inhibit expression or activity of Ga12, Ga13, RhoA, and ROCK, respectively. Cells that were scratched with tips were then stimulated with gastrin. Representative images from left to right indicate the Golgi marker protein $\beta$-cop (red), cytoskeletal F-actin (green), and cell nucleus (blue). Merged images and wind rose plots of Golgi orientation are shown. Gastrin promoted the reorganization and polarization of the Golgi apparatus at the leading edge of PANC-1 cells facing the detached line; however, a highly disorganized and fragmented Golgi apparatus was distributed in the cytoplasm. Gastrin also induced the polarization of cytoskeletal F-actin accompanied with a non-polarized morphology. Ga12 RNAi, Ga13 RNAi, Rosin, and Y27632 pre-treatment blocked the polarization of the Golgi and F-actin that was induced by gastrin. Wind rose plots indicate the Golgi orientation in cells, and only the gastrin treatment group restored proper cell polarity and Golgi orientation

characterized. As mentioned above, we have shown that Ga12/13-RhoA-ROCK signaling is crucial for the morphological changes and high metastasis potential of gastrin-induced cells. We then tried to delineate the role of Go12/13-RhoA-ROCK signaling in reorientation of the Golgi apparatus. We used a scratch wound healing model to induce the migration of PANC-1 cells and utilized a $\beta$-COP antibody to mark the location of the Golgi apparatus. As shown in the immunofluorescence and wind rose plot (Fig. 5), $200 \mathrm{nM}$ gastrin treatment for $6 \mathrm{~h}$ significantly promoted the reorientation of the Golgi apparatus to the leading edge of the cells; however, in the DMSO group, the Golgi apparatus was disordered with no directivity. We then individually treated the cells with Go12 siRNA, Go13 siRNA, Rhosin, or Y27632, and we demonstrated that any missing link in Go12/ 13-RhoA-ROCK signaling impaired the polarization of F-actin and impeded the reorientation of the Golgi in response to wounding. These results reveal a previously unidentified pathway that regulates the polarity of migrating cancer cells through Golgi reorientation.

\section{Gastrin promotes progression of pancreatic cancer in nude mice with orthotopic transplantation}

Next, we tested whether gastrin is capable of driving progression of pancreatic cancer cells in vivo. We transplanted PANC-1 cells under the pancreas envelope in nude mice. During the experiment, mice in different groups were administered different treatments (Fig. 6a). After 7 weeks, the mice were killed. Western blot analysis and immunohistochemistry staining showed that, compared with the control group (DMSO treatment), paxillin activation in the orthotopic tumor was significantly enhanced in the gastrin treatment group. YM022, a specific blocker of the CCKBR, and Y27632 reduced the phosphorylation level of paxillin induced by gastrin (Fig. 6b, c). Consistent with the results of western blot analysis, the weight of orthotopic tumors in the gastrin treatment group was the highest and YM022 and Y27632 inhibited the growth of the tumors (Fig. 6c, d).

Pancreatic cancer spreads easily to distant organs, and the liver is its main target organ ${ }^{27}$; therefore, we checked the number of metastasis sites in the liver of the treated mice. As expected, gastrin significantly promoted the metastasis of pancreatic cancer cells, and YM022 and Y27632 inhibited this process (Fig. 6e). From these results, we further confirm that gastrin can promote pancreatic cancer progression via the CCKBR and its downstream signaling pathway.

\section{Discussion}

In this study, we demonstrated that gastrin promotes the reorientation of the Golgi apparatus and directional migration of pancreatic cancer cells by inducing the activation of paxillin and FAK via the CCKBR-G $\alpha 12$ / 13-RhoA-ROCK signaling pathway (as illustrated in Fig. 7).

The functions of gastrin and the CCKBR in cancer biology have been investigated and discussed for decades. It has been demonstrated that the downstream signaling pathway of the gastrin/CCKBR includes JNK, JAK2, STAT3, ERKs, and NF-kappaB ${ }^{28-30}$. In pancreatic cancer, gastrin induces the upregulation of beta1-integrin via the Src family of kinases and the PI3K pathway, resulting in enhanced invasion ability ${ }^{31}$. Further, $\alpha v$ integrin is upregulated by gastrin in pancreatic cancer cells and contributes to cell adhesion ${ }^{32}$. It has also been reported that gastrin regulates the oncogenic protein ABCG2 via NFkappaB signaling in pancreatic cancer ${ }^{30}$. However, the roles of gastrin and the CCKBR in cellular directional migration had not been investigated. To our best knowledge, this study was the first to discover that gastrin can induce directional migration of cancer cells. Our research provides a supplementary explanation for how aberrant enrichment of gastrin in the tumor microenvironment and peripheral blood affects the morphological changes and invasion ability of malignant cells.

Reorientation of the Golgi apparatus is a key characteristic of the directional migration of cells ${ }^{7,8}$. Importantly, we observed that the location of the Golgi apparatus was responsive to gastrin. As we know, the Golgi apparatus is crucial for protein processing and membrane dynamics, so our results indicate that gastrin might promote cancer metastasis by facilitating polarized 

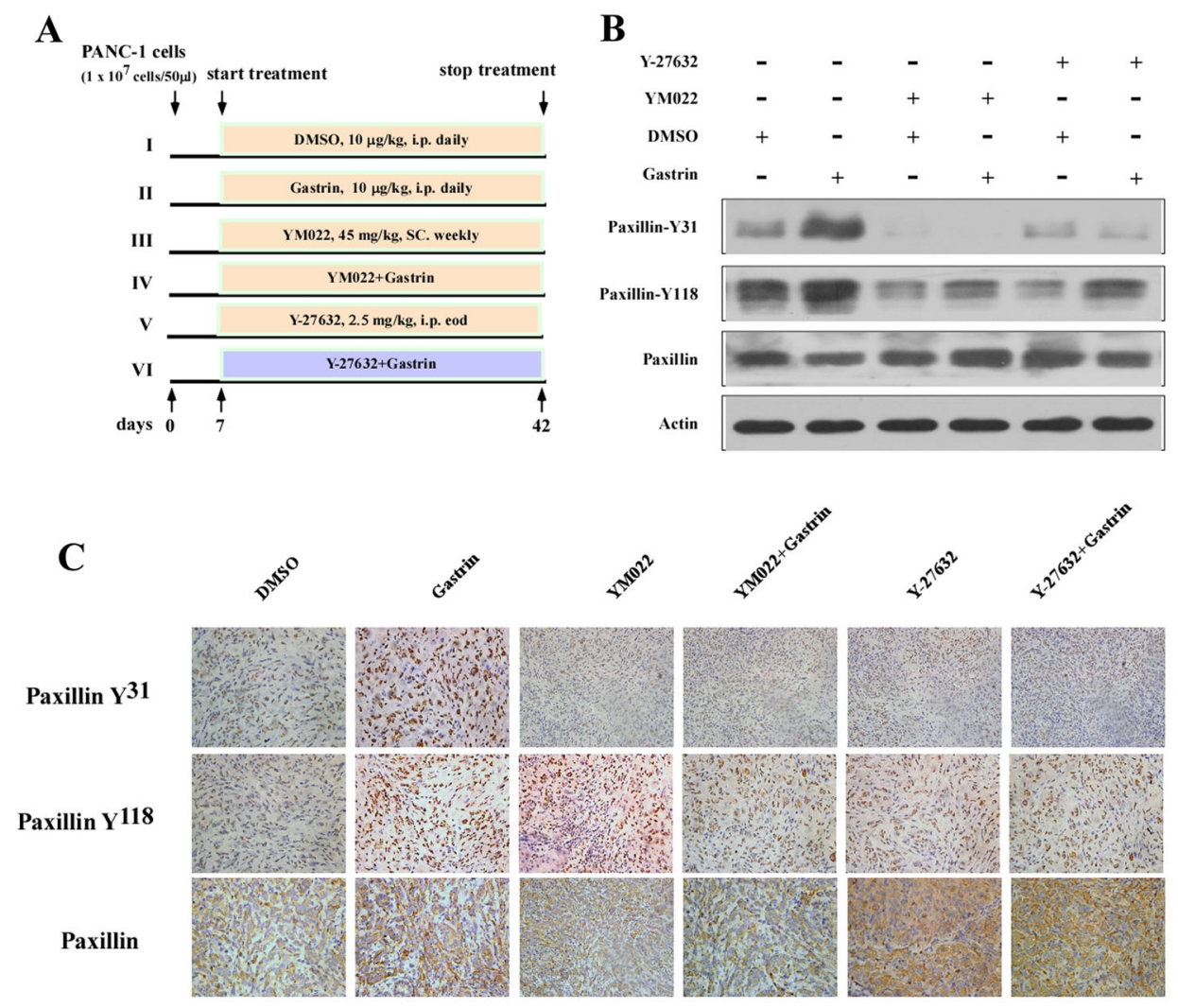

D
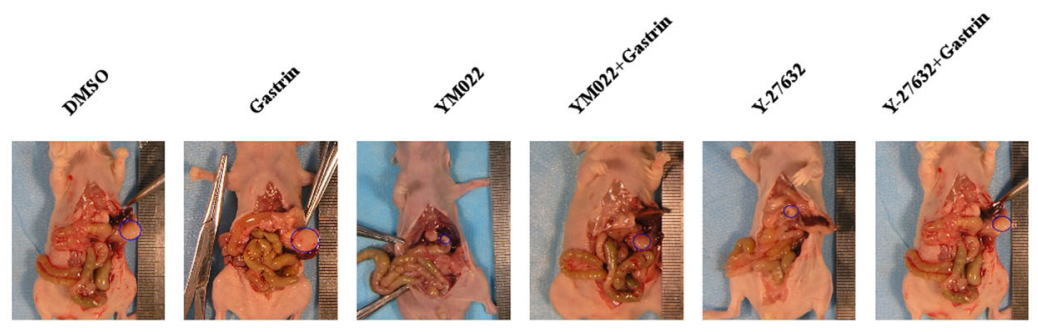

$\mathbf{E}$
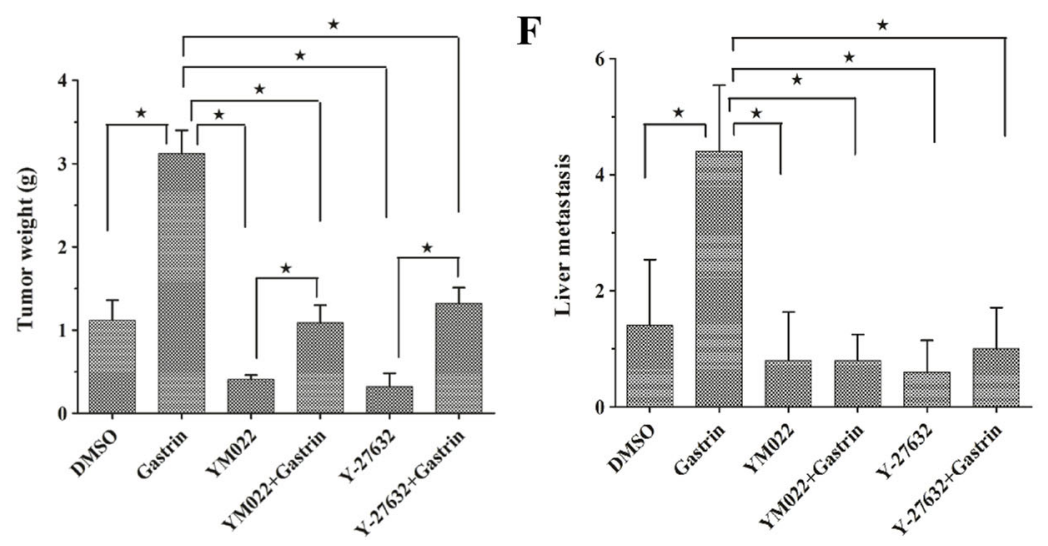

Fig. 6 Gastrin promotes tumor progression of orthotopic tumors in nude mice. PANC-1 cells $(1 \times 107$ cells $)$ were injected into the head/neck region of the nude mouse pancreas. a Mice were divided into six groups and treated according to schedule. b, c Immunoblotting (b) and immunohistochemistry (c) results are indicated for paxillin phosphorylation of orthotopic pancreatic cancer tissues. $\mathbf{d}-\mathbf{f}$ YM022 and Y-27632 significantly inhibited the growth of the primary pancreatic tumor $(\mathbf{d}, \mathbf{e})$ and prevented liver metastasis $(\mathbf{f})$. Data are represented as the mean $\pm S D, * P$ $<0.05$ 


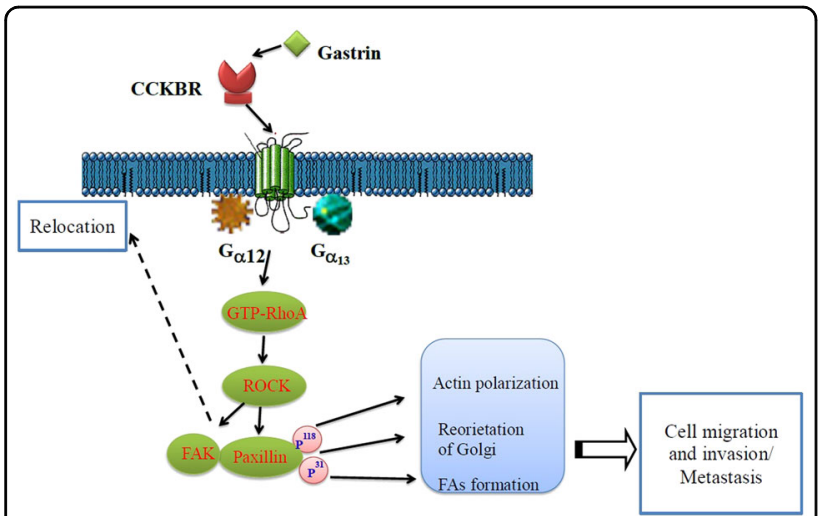

Fig. 7 Schematic model depicting the role of gastrin-CCKBR-Ga12/ 13-RhoA-ROCK signaling in pancreatic cancer progression

secretion and providing membrane to sites of directional migration. The molecular events that modulate the reorientation of the Golgi during cell migration have not been determined. Studies have shown that repositioning of the Golgi results from the reorganization of the microtubule $^{33,34}$. Among the regulators of the cytoskeleton, the Rho family of small GTPases is principle ${ }^{24}$. Our results showed that in pancreatic cancer, Golgi reorientation, which is accompanied by $\mathrm{F}$-actin reorganization, is induced by gastrin and is due to activation of RhoA-ROCK signaling. Our findings are consistent with previously established ideas.

During the formation of focal adhesion, paxillin is an adapter protein that functions in an important scaffolding role by recruiting structural and signaling molecules when it is phosphorylated on specific Tyr and Ser residues ${ }^{18,35,36}$. Among these residues, Tyr31 and Tyr118 are the most important ${ }^{18}$. Paxillin phosphorylation results in the generation of specific $\mathrm{SH} 2$ and $\mathrm{SH} 3$ interaction sites that make a link between integrin receptors and cytoskeletal elements, such as actin and vinculin ${ }^{37,38}$. Additionally, paxillin interacts with and facilitates the phosphorylation of FAK, which is important for binding to downstream signaling molecules, such as Src, PI3K, and $\mathrm{SHC}^{18,39}$. Mutation, amplification, overexpression, and aberrant activation of paxillin have been involved in many cancers. Paxillin has been associated with proliferation, chemoresistance, and epithelial-mesenchymal transition in cancer research $^{40-42}$. Diverse stimuli can induce paxillin phosphorylation, such as Src and FAK ${ }^{18,43}$. Previously, we demonstrated that in human colon cancer cells, gastrininduced tyrosine phosphorylation of paxillin in a time- and dose-dependent manner ${ }^{20}$, while its mechanism is obscure. Herein, we found that gastrin-activated paxillin through the Go12/13-RhoA-ROCK signaling pathway, which provides a reasonable explanation.
The heterotrimeric $G$ proteins $G \alpha 12$ and $G \alpha 13$ are widely expressed in human tissues, have been described as RhoA activators via RH-containing RhoGEFs, and can promote actin stress fiber formation and focal adhesion assembly ${ }^{22,23}$. Ga12/13 have consistently been demonstrated to play a key role in oncogenic transformation in multiple cancers, such as prostate cancer, breast cancer, and cervical cancer ${ }^{44-46}$, while its role in pancreatic cancer is largely unknown. However, another main finding of this current work is that Go12/13 are responsible for gastrin's function in pancreatic cancer. Intriguingly, as shown in Fig. 2a, b, compared with the control group, Go12 knockdown resulted in reduction of the basic levels of phosphorylated paxillin and activated RhoA (Go13 knockdown only reduced the basic levels of phosphorylated paxillin, Supplementary Figure 2A-B), and this indicated that $G \alpha 12 / 13$ also modulated endogenous or alternative activation of paxillin and RhoA in pancreatic cancer. This result suggested that Go12/13 may be potential therapy targets for pancreatic cancer.

Target therapy has been successful in a number of malignancies. Unfortunately, the results of pancreatic cancer treatments have been extremely disappointing to date. The GPCR family, with over 800 members, is the largest and most diverse protein family in the human proteome ${ }^{47}$. GPCRs play important roles in numerous cellular and physiological processes, and consequently, aberrant receptor expression or activity is demonstrated in many disorders and diseases ${ }^{47}$. Importantly, GPCRs are considered to be druggable proteins, and have been the most successful pharmaceutical target class in cancer therapy. For example, degarelix targets $\mathrm{GnRH}$ in prostate cancer $^{48}$, sonidegib and vismodegib target SMO in basal cell carcinoma ${ }^{49,50}$, and mogamulizumab targets CCR4 in lymphoma ${ }^{51}$. Previous studies have demonstrated that gastrin and its receptor, CCKBR, are involved in the tumorigenesis and progression of pancreatic cancer. CCKBR, a GPCR member, has been regarded as a promising target in the therapy of pancreatic adenocarcinoma ${ }^{10}$. The CCKBR antagonists JB95008 and Z-360 have been tested in clinical trial, but the results have been unsatisfactory $^{52,53}$. Our research elucidated a key signaling pathway in pancreatic cancer progression mediated by gastrin and/or CCKBR. Hopefully, our findings can provide clues for novel CCKBR targeting-drug development and can improve the prognosis of pancreatic cancer patients.

\section{Acknowledgements}

This study was supported by grants from the Fundamental Research Funds for the Chinese Central Universities (no. 2012302020214), National Natural Science Foundation of China (nos. 81602116, 81672387, and 81703030) and Hubei Provincial Natural Science Foundation (no. 2016CFB195). 


\section{Author details}

${ }^{1}$ Department of Gastroenterology, Renmin Hospital of Wuhan University, Wuhan 430060, China. ${ }^{2}$ Key Laboratory of Hubei Province for Digestive System Disease, Renmin Hospital of Wuhan University, Wuhan, China. ${ }^{3}$ School of Electrical and Electronic Engineering, Nanyang Technological University, Singapore, Singapore. ${ }^{4}$ Department of Anatomical and Cellular Pathology, State Key Laboratory of Oncology in South China, Prince of Wales Hospital, The Chinese University of Hong Kong, Hong Kong, China. ${ }^{5}$ Department of Gastroenterology, The First Affiliated Hospital of Zhengzhou University, Zhengzhou, China. 'Department of General Surgery, The Second People's Hospital of Guangdong Province, Southern Medical University, Guangzhou, China

\section{Conflict of interest}

The authors declare that they have no conflict of interest.

\section{Publisher's note}

Springer Nature remains neutral with regard to jurisdictional claims in published maps and institutional affiliations.

Supplementary information accompanies this paper at https://doi.org/ 10.1038/s12276-018-0081-6.

Received: 11 November 2017 Revised: 2 February 2018 Accepted: 5 February 2018.

Published online: 1 May 2018

\section{References}

1. Torre, L. A. et al. Global cancer statistics, 2012. CA Cancer J. Clin. $\mathbf{6 5}, 87-108$ (2015).

2. Gaianigo, N., Melisi, D. \& Carbone C. EMT and treatment resistance in pancreatic cancer. Cancers 9, E122 (2017).

3. Mizoguchi, T., Ikeda, S., Watanabe, S., Sugawara, M. \& Itoh, M. Mib1 contributes to persistent directional cell migration by regulating the Ctnnd1-Rac1 pathway. Proc. Natl Acad. Sci. USA 114, e9280-e9289 (2017).

4. Grande-García, A. \& del Pozo, M. A. Caveolin-1 in cell polarization and directional migration. Eur. J. Cell Biol. 87, 641-647 (2008).

5. Yuan, X. et al. Directional migration in esophageal squamous cell carcinoma (ESCC) is epigenetically regulated by SET nuclear oncogene, a member of the inhibitor of histone acetyltransferase complex. Neoplasia 19, 868-884 (2017).

6. Lupo, B. et al. Tankyrase inhibition impairs directional migration and invasion of lung cancer cells by affecting microtubule dynamics and polarity signals. BMC Biol. 14, 5 (2016).

7. Mardakheh, F. K., Self, A. \& Marshall, C. J. RHO binding to FAM65A regulates Golgi reorientation during cell migration. J. Cell Sci. 129, 4466-4479 (2016).

8. Xing, M. et al. GOLPH3 drives cell migration by promoting Golgi reorientation and directional trafficking to the leading edge. Mol. Biol. Cell 27, 3828-3840 (2016).

9. Wank, S. A. G protein-coupled receptors in gastrointestinal physiology. I. CCK receptors: an exemplary family. Am. J. Physiol. 274, G607-G613 (1998).

10. Boyce, M., Lloyd, K. A. \& Pritchard, D. M. Potential clinical indications for a CCK2 receptor antagonist. Curr. Opin. Pharmacol. 31, 68-75 (2016).

11. Masià-Balagué, M. et al. Gastrin-stimulated Ga13 activation of Rgnef protein (ArhGEF28) in DLD-1 colon carcinoma cells. J. Biol. Chem. 290, 15197-15209 (2015).

12. Smith, J. P., Hamory, M. W., Verderame, M. F. \& Zagon, I. S. Quantitative analysis of gastrin mRNA and peptide in normal and cancerous human pancreas. Int. J. Mol. Med. 2, 309-315 (1998).

13. Smith, J. P., Shih, A., Wu, Y., McLaughlin, P. J. \& Zagon, I. S. Gastrin regulates growth of human pancreatic cancer in a tonic and autocrine fashion. Am. J. Physiol. 270, R1078-R1084 (1996).

14. Li, C. et al. The GTPase Rab43 controls the anterograde ER-Golgi trafficking and sorting of GPCRs. Cell Rep. 21, 1089-1101 (2017).

15. Donnelly, S. K., Bravo-Cordero, J. J. \& Hodgson, L. Rho GTPase isoforms in cell motility: don't fret, we have FRET. Cell Adh. Migr. 8, 526-534 (2014).

16. Stepan, V. et al. Role of small GTP binding proteins in the growth-promoting and antiapoptotic actions of gastrin. Am. J. Physiol. Gastrointest. Liver Physiol. 287, G715-G725 (2004).
17. Le Page, S. L., Bi, Y. \& Williams, J. A. CCK-A receptor activates RhoA through G alpha 12/13 in NIH3T3 cells. Am. J. Physiol. Cell Physiol. 285, C1197-C1206 (2003).

18. López-Colomé, A. M., Lee-Rivera, l., Benavides-Hidalgo, R. \& López, E. Paxillin: a crossroad in pathological cell migration. J. Hematol. Oncol. 10, 50 (2017).

19. Leyton, J., Garcia-Marin, L. J., Tapia, J. A., Jensen, R. T. \& Moody, T. W. Bombesin and gastrin releasing peptide increase tyrosine phosphorylation of focal adhesion kinase and paxillin in non-small cell lung cancer cells. Cancer Lett. 162, 87-95 (2001).

20. Yu, H. G., Schrader, H., Otte, J. M., Schmidt, W. E. \& Schmitz, F. Rapid tyrosine phosphorylation of focal adhesion kinase, paxillin, and p130Cas by gastrin in human colon cancer cells. Biochem. Pharmacol. 67, 135-146 (2004).

21. Suzuki, N., Hajicek, N. \& Kozasa, T. Regulation and physiological functions of G12/13-mediated signaling pathways. Neurosignals 17, 55-70 (2009).

22. He, Z. et al. CYP2J2 metabolites, epoxyeicosatrienoic acids, attenuate Ang IIinduced cardiac fibrotic response by targeting Ga12/13. J. Lipid Res. 58, 1338-1353 (2017).

23. Xie, S., Mason, F. M. \& Martin, A. C. Loss of Ga12/13 exacerbates apical area dependence of actomyosin contractility. Mol. Biol. Cell 27, 3526-3536 (2016).

24. Marjoram, R. J., Lessey, E. C. \& Burridge, K. Regulation of RhoA activity by adhesion molecules and mechanotransduction. Curr. Mol. Med. 14, 199-208 (2014).

25. Shi, Y. et al. The mDial formin is required for neutrophil polarization, migration, and activation of the LARG/RhoA/ROCK signaling axis during chemotaxis. J. Immunol. 182, 3837-3845 (2009).

26. Cao, X. et al. A phosphorylation switch controls the spatiotemporal activation of Rho GTPases in directional cell migration. Nat. Commun. 6, 7721 (2015).

27. Ito, $T$. et al. The diagnostic advantage of EOB-MR imaging over $C T$ in the detection of liver metastasis in patients with potentially resectable pancreatic cancer. Pancreatology 17, 451-456 (2017).

28. Beales, I. L. \& Ogunwobi, O. Glycine-extended gastrin inhibits apoptosis in colon cancer cells via separate activation of Akt and JNK pathways. Mol. Cell Endocrinol. 247, 140-149 (2006).

29. $\mathrm{Xu}, \mathrm{W}$. et al. Gastrin acting on the cholecystokinin2 receptor induces cyclooxygenase-2 expression through JAK2/STAT3/PI3K/Akt pathway in human gastric cancer cells. Cancer Lett. 332, 11-18 (2013).

30. Wang, J. et al. Gastrin regulates $A B C G 2$ to promote the migration, invasion and side populations in pancreatic cancer cells via activation of NF-KB signaling. Exp. Cell Res. 346, 74-84 (2016).

31. Cayrol, C. et al. Cholecystokinin-2 receptor modulates cell adhesion through beta 1-integrin in human pancreatic cancer cells. Oncogene 25, 4421-4428 (2006).

32. Cayrol, C. et al. $v$ integrin: a new gastrin target in human pancreatic cancer cells. World J. Gastroenterol. 17, 4488-4495 (2011).

33. Jiang, P. et al. The protein encoded by the CCDC170 breast cancer gene functions to organize the Golgi-microtubule network. EBio Med. 22, 28-43 (2017).

34. Wesolowski, J. et al. Chlamydia hijacks ARF GTPases to coordinate microtubule posttranslational modifications and Golgi complex positioning. MBio 8 , e02280-16 (2017).

35. Fusté, N. P. et al. Cytoplasmic cyclin D1 regulates cell invasion and metastasis through the phosphorylation of paxillin. Nat. Commun. 7, 11581 (2016).

36. Qin, R. et al. Phosphorylation and turnover of paxillin in focal contacts is controlled by force and defines the dynamic state of the adhesion site. Cytoskeleton 72, 101-112 (2015).

37. Turner, C. E. \& Miller, J. T. Primary sequence of paxillin contains putative $\mathrm{SH} 2$ and SH3 domain binding motifs and multiple LIM domains: identification of a vinculin and pp125Fak-binding region. J. Cell Sci. 107, 1583-1591 (1994).

38. Deakin, N. O., Ballestrem, C. \& Turner, C. E. Paxillin and Hic-5 interaction with vinculin is differentially regulated by Rac1 and RhoA. PLoS ONE 7, e37990 (2012).

39. Wade, R. \& Vande Pol, S. Minimal features of paxillin that are required for the tyrosine phosphorylation of focal adhesion kinase. Biochem. J. 393, 565-573 (2006).

40. Zhang, L. L. et al. Phosphatase and tensin homolog (PTEN) represses colon cancer progression through inhibiting paxillin transcription via PI3KAKT/NF-KB pathway. J. Biol. Chem. 290, 15018-15029 (2015).

41. Kanteti, R., Batra, S. K., Lennon, F. E. \& Salgia, R. FAK and paxillin, two potential targets in pancreatic cancer. Oncotarget 7, 31586-31601 (2016).

42. Wu, D. W., Huang, C. C., Chang, S. W., Chen, T. H. \& Lee, H. Bcl-2 stabilization by paxillin confers 5 -fluorouracil resistance in colorectal cancer. Cell Death Differ. 22, 779-789 (2015). 
43. Kratimenos, P. et al. FAK-Src-paxillin system expression and disease outcome in human neuroblastoma. Pediatr. Hematol. Oncol. 34, 221-230 (2017).

44. Kelly, P. et al. A role for the $\mathrm{G} 12$ family of heterotrimeric $\mathrm{G}$ proteins in prostate cancer invasion. J. Biol. Chem. 281, 26483-26490 (2006).

45. Juneja, J., Cushman, I. \& Casey, P. J. G12 signaling through c-Jun NH2-terminal kinase promotes breast cancer cell invasion. PLOS ONE 6, e26085 (2011).

46. Yuan, B., Cui, J., Wang, W. \& Deng, K. Ga12/13signaling promotes cervical cancer invasion through the RhoA/ROCK-JNK signaling axis. Biochem. Biophys. Res. Commun. 473, 1240-1246 (2016).

47. Nieto Gutierrez, A. \& McDonald, P. H. GPCRs: emerging anti-cancer drug targets. Cell Signal. 41, 65-74 (2018).

48. Sayyid, R. K. et al. A phase II, randomized, open-label study of neoadjuvant degarelix versus LHRH agonist in prostate cancer patients prior to radical prostatectomy. Clin. Cancer Res. 23, 1974-1980 (2017).
49. Casey, D. et al. FDA approval summary: sonidegib for locally advanced basal cell carcinoma. Clin. Cancer Res. 23, 2377-2381 (2017).

50. Basset-Séguin, N. et al. Vismodegib in patients with advanced basal cell carcinoma: primary analysis of STEVIE, an international, open-label trial. Eur. J. Cancer 86, 334-348 (2017).

51. Makita, S. \& Tobinai, K. Mogamulizumab for the treatment of T-cell lymphoma. Expert Opin. Biol. Ther. 17, 1145-1153 (2017).

52. Chau, I. et al. Gastrazole (JB95008), a novel CCK2/gastrin receptor antagonist, in the treatment of advanced pancreatic cancer: results from two randomised controlled trials. Br. J. Cancer 94, 1107-1115 (2006).

53. Ueno, M. et al. A randomized phase II study of gemcitabine plus Z-360, a CCK2 receptor-selective antagonist, in patients with metastatic pancreatic cancer as compared with gemcitabine plus placebo. Cancer Chemother. Pharmacol. 80, 307-315 (2017). 